\title{
ARTICLE
}

Received 10 Jul 2014 | Accepted 21 Jan 2015 | Published 27 Feb 2015 DOl: 10.1038/ncomms7343

\section{Narrowband light detection via internal quantum efficiency manipulation of organic photodiodes}

\author{
Ardalan Armin ${ }^{1}$, Ross D. Jansen-van Vuuren¹, Nikos Kopidakis², Paul L. Burn ${ }^{1} \&$ Paul Meredith ${ }^{1}$
}

Spectrally selective light detection is vital for full-colour and near-infrared (NIR) imaging and machine vision. This is not possible with traditional broadband-absorbing inorganic semiconductors without input filtering, and is yet to be achieved for narrowband absorbing organic semiconductors. We demonstrate the first sub-100 nm full-width-at-half-maximum visible-blind red and NIR photodetectors with state-of-the-art performance across critical response metrics. These devices are based on organic photodiodes with optically thick junctions. Paradoxically, we use broadband-absorbing organic semiconductors and utilize the electro-optical properties of the junction to create the narrowest NIR-band photoresponses yet demonstrated. In this context, these photodiodes outperform the encumbent technology (input filtered inorganic semiconductor diodes) and emerging technologies such as narrow absorber organic semiconductors or quantum nanocrystals. The design concept allows for response tuning and is generic for other spectral windows. Furthermore, it is materialagnostic and applicable to other disordered and polycrystalline semiconductors.

\footnotetext{
${ }^{1}$ Centre for Organic Photonics \& Electronics, School of Mathematics and Physics and School of Chemistry and Molecular Biosciences, The University of Queensland, Brisbane, Queensland 4072, Australia. ${ }^{2}$ National Renewable Energy Laboratory, Golden, Colorado 80401, USA. Correspondence and requests for materials should be addressed to P.L.B. (email: p.burn2@uq.edu.au) or P.M. (email: meredith@physics.uq.edu.au).
} 
$\mathrm{P}$ hotodiodes are the most common photodetector architecture and are generally classified as broadband (panchromatic) or narrowband (wavelength selective), depending on their spectral responsivity bandwidth ${ }^{\mathrm{P}}$. In general, for broadband systems it is desirable to have a spectrally flat, high external quantum efficiency (EQE) across the wavelength range of interest ${ }^{2}$. Broadband photodiodes are used predominantly in multi-colour photodetection under low light conditions (for example, in day-or-night surveillance)-essentially operating as integrating detectors. For narrowband applications such as fullcolour imaging or visible-blind near-infrared (NIR) detection (for example in light curtains, security systems and intelligent monitoring), spectral (colour) discrimination is required. This can be achieved with one of two possible approaches: the use of a broadband detector in combination with a dichroic prism or set of optical filters at the input side (we refer to this as input filtering); or the use of a truly narrowband detector with photoresponsive semiconductors engineered for the desired spectral window. The former option has been the predominant strategy for inorganic semiconductor-based narrowband photodetection ${ }^{3}$. However, such a strategy complicates the design of the image sensor and can also lead to degradation of the picture quality and colour constancy ${ }^{4-8}$. The molecular nature of organic semiconductors means that organic photodiodes (OPDs) can be designed with relatively narrow spectral responses of $<200 \mathrm{~nm}$ full-width-at-half-maximum (FWHM) and respectable EQEs (10-30\%), but the suppression of response outside the chosen spectral window still remains a significant constraint $^{8}$. For certain imaging applications such as machine vision systems, the ideal case is a quasi-Gaussian spectral response with a $\mathrm{FWHM} \leq 100 \mathrm{~nm}$ that does not give a response (that is, 'blind') outside the chosen window'.

There is growing interest in the development of new materials and OPD architectures that selectively detect light in the red $(620-750 \mathrm{~nm})$ or NIR ${ }^{2,10}$. Red light photodetectors are useful for short-distance optical communications ${ }^{11}$ and they also form a critical component in three-and-four-colour photosensors 9,12 . The best examples have FWHMs of $\sim 200 \mathrm{~nm}$ but with significant and undesirable blue and green sensitivity $8,13,14$. NIR photodetectors are important in medical, industrial, communication, scientific and military applications as well as for night-vision imaging and security ${ }^{14-16}$. NIR-sensitive photodectectors have previously been demonstrated using inorganic quantum dot sensitization ${ }^{17,18}$, narrow optical-gap donor organic semiconductors within a conventional thin heterojunction structure ${ }^{14,19,20}$, and more recently by harvesting sub-gap charge transfer states $(\mathrm{CT})^{15,21,22}$. Yang et al. have reported on the use of CT state absorption to extend the spectral response of an OPD based on a P3HT:PC60BM blend to the $\mathrm{NIR}^{22}$. It is important to note that this is not a particularly efficient method to produce a NIR response since the CT state absorption is very weak in organic semiconductors ${ }^{21}$ and therefore extremely thick junctions $(>10 \mu \mathrm{m})$ are required for light harvesting via CT states. As a consequence, the OPDs of Yang et al. ${ }^{22}$ could only be operated at extremely large reverse bias voltages $(>100 \mathrm{~V})$, and they also had a very high dark current noise. A truly narrowband (FWHM $<100 \mathrm{~nm}$ ), low noise, visible-blind NIR detector is yet to be realized.

The aforementioned thin heterojunction architecture has become the standard design for OPDs. The heterojunction contains two components: an electron acceptor and electron donor, akin to $n$ - and $p$-type inorganic semiconductors, respectively. The acceptor and donor are either blended at the molecular scale to form a bulk heterojunction $(\mathrm{BHJ})^{23}$ or deposited sequentially to form a planar heterojunction ${ }^{24}$. In both cases the photo-active junction is typically of the order 10 to
$100 \mathrm{~nm}$ in thickness, and is sandwiched between anode and cathode electrodes (one of which is semi-transparent) and various blocking or transport layers. In these heterojunctions, careful attention must be given to the optical absorption of the acceptor and donor since both can contribute to the photocurrent through the so-called photo-induced electron (Channel I) and hole transfer (Channel II) processes ${ }^{25}$. While a large range of electron donor materials are available $6,8,15,19$, the choice of electron acceptor is somewhat limited and is dominated by the fullerenes such as PC60BM $\left([6,6]\right.$-phenyl- $\mathrm{C}_{60}$-butyric acid methyl ester) and PC70BM ([6,6]-phenyl- $\mathrm{C}_{70}$-butyric acid methyl ester). ${ }^{1}$ A relatively small number of non-fullerene acceptors have been reported but very few exhibit acceptable quantum efficiencies within organic photovoltaic cells ${ }^{26}$. This is problematic for narrowband red or NIR OPDs since the fullerenes all possess significant blue-green absorption-considerably broadening the overall device response and/or rendering the detector non-blind in the visible ${ }^{14,27}$. The best narrowband red and NIR OPDs to date are based on engineering the absorption spectrum of the materials, rather than a broadband junction plus filter or prism designs $^{8,28}$.

Conventional heterojunction OPDs are thin film, low finesse cavities $^{29}$. As such, the EQE of the diode is dictated by the absorption of the junction components modified by interference effects derived from multiple reflections between the metallic and semi-transparent electrodes. These micro-cavity phenomena can act to broaden or narrow certain spectral features ${ }^{30}$. Understanding this physics provides additional tools for response tuning-varying the thickness of the photo-active layer $^{31}$ or the use of optical spacers to control the micro-cavity effects $^{32}$. However, the strong blue-green fullerene absorption prevents the design of a single-mode red or NIR cavity.

Harrison et al. ${ }^{33}$ have shown that a narrow response can be obtained around the absorption onset of neat MEH-PPV thick photodiodes via enhanced exciton dissociation efficiency at lower energies. In our current work, we introduce the new concept of charge collection narrowing (CCN) in thick bulk heterojunctions to achieve narrowband OPDs. The CCN devices operate based on narrowing the charge collection efficiency to the desired spectral region to overcome the above-mentioned limitations. We thus report the first sub-100 nm FWHM, visible-blind red and NIR photodetectors. Furthermore, the use of thick junctions markedly decreases the defect density ${ }^{34}$ and therefore significantly suppresses the dark current ${ }^{35}$, resulting in high specific detectivities $\left(\sim 10^{12}\right.$ Jones) and a large linear dynamic range (LDR)- in this case 8 orders of magnitude. Moreover, we demonstrate that these CCN OPDs can be spectrally fine-tuned over a range of $\sim 100 \mathrm{~nm}$. This methodology is generic and the detection window can be chosen depending on the absorption onset of the junction materials.

\section{Results}

Spectral shape of the EQE. OPDs utilizing thick junctions have previously been reported $15,22,33,35$. In particular, Armin et al. ${ }^{35}$ recently described photodiodes with impressive performance metrics for broadband visible, IR-blind light detection using the archetypal bulk heterojunction organic semiconductor donor: acceptor blend of PCDTBT:PC70BM [(poly $\left[N-9^{\prime \prime}\right.$-heptadecanyl2,7-carbazole-alt-5,5-( $4^{\prime}, 7^{\prime}$-di-2-thienyl-2' ${ }^{\prime}, 1^{\prime}, 3^{\prime}$-benzothiadiazole $\left.)\right]$ : PC70BM]. The quantum efficiency of these broadband devices was spectrally flat because efficient 'volume generated' charge carrier collection was achieved for all visible band wavelengths. In the current work, we utilize a completely different junction thickness regime $(>1.5 \mu \mathrm{m})$ in combination with the CCN concept to produce the aforementioned narrowband spectral 
responses. It is important to note that our red narrowband devices utilize the same donor:acceptor combination (PCDTBT: PC70BM) as the broadband devices of Armin et $a l^{35}$. This shows that $\mathrm{CCN}$ is a device physics-related concept that delivers a new way of controlling spectral response over-and-above that afforded by the optical properties of the junction materials. As will be described later, the overall effect is to shape the internal quantum efficiency (IQE) to obtain spectral selectivity by markedly misbalancing the charge carrier transport. This is in stark contrast to any previously reported thick junction OPDs.

To understand the CCN concept, we begin with the definition of the EQE which is generally given by ${ }^{36}$ :

$$
\operatorname{EQE}(\lambda)=\eta_{\mathrm{abs}}(\lambda) \eta_{\text {gen }} \eta_{\text {coll }}=\eta_{\mathrm{abs}}(\lambda) \cdot \mathrm{IQE},
$$

Where, $\eta_{\mathrm{abs}}$ is the light absorption within the photo-active layer (the heterojunction), $\eta_{\text {gen }}$ is the charge carrier generation quantum yield via exciton and CT state dissociation, and $\eta_{\text {coll }}$ is the photo-generated charge carrier collection efficiency. The IQE is the ratio of the EQE to the number of absorbed photons and can be spectrally flat in conventional photodiodes as shown in recent studies ${ }^{21,29}$. This means that the IQE does not influence the spectral sensitivity unless there are additional energydependent phenomena operating such as hot excitons ${ }^{37}$ or marked differences in the Channel I and Channel II charge generation efficiencies ${ }^{38}$.

Conventional thin film bulk heterojunction OPDs. The working principles of conventional thin film bulk heterojunction and CCN OPDs are presented schematically in Fig. 1a-d, which show the device structure, photo-generated carrier distribution profile, and the EQE and absorption spectra of the photo-active layers, respectively. Although the figures are schematics for illustrative purposes, knowledge of the optical constants ( $n$ and $k$ see Methods) of all layers in the diode structure allows one to accurately simulate the actual optical field distributions, EQE and absorption using a transfer matrix analysis ${ }^{29,39,40}$. In this case the PCDTBT:PC70BM optical constants are used to demonstrate the concept.

As shown in Fig. 1a, in a typical thin $(\sim 100 \mathrm{~nm})$ junction OPD, the shape of the optical field distributions derived from the absorbed photons are somewhat independent of the incident light wavelength (for example, $\mathbf{A}, \mathbf{B}$ and $\mathbf{C}$ ). In this figure, $\mathbf{A}$ and $\mathbf{B}$ are wavelengths above the optical gap, while $\mathbf{C}$ is near the absorption edge of the blend ${ }^{21}$. All three wavelengths produce volume photocarrier generation, and, given the 'thinness' of the junction, one would not expect any significant spectral dependence of the charge carrier collection efficiency, $\eta_{\text {coll }}$, since the drift distances for carriers generated at the three variable wavelengths are approximately the same. This feature of volume photo-carrier generation in thin junctions has been confirmed by spectral and field independent IQE and charge photogeneration measurements in thin junctions ${ }^{41,42}$. In the absence of any exotic energy dependence of charge generation and transport, the EQE spectrum follows the spectral shape of the light absorption within the active layer. Therefore, one method to achieve a narrowband spectral response is to use narrowband absorbing organic semiconductors in a carefully designed junction (cavity) structure $^{30}$.

b

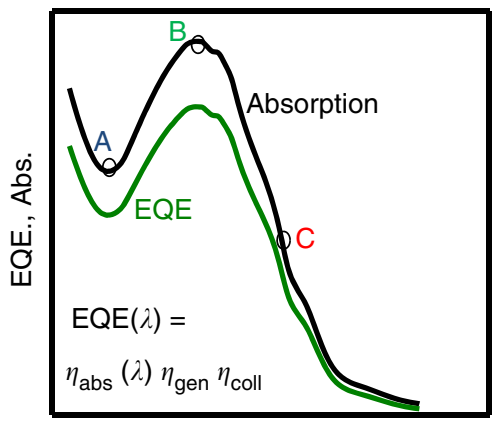

Wavelength

d

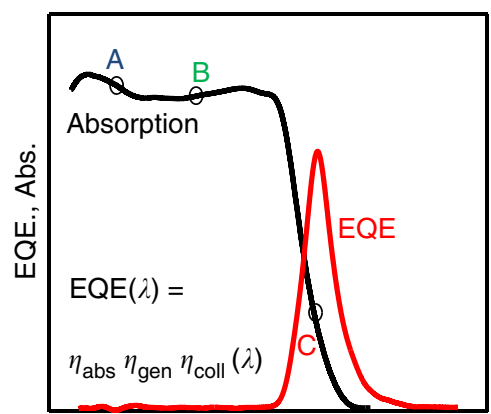

Wavelength

Figure 1 | Working principles of conventional and CCN OPDs. The photodiode structures are shown with the absorbed photon distribution for selected wavelengths marked on the absorption spectra within the active layer (A, B and $\mathbf{C}$ ). Conventional narrowband OPD (a) and (b): in this case, photoexcitations are distributed within the active layer volume for all three wavelengths $\mathbf{A}, \mathbf{B}$ and $\mathbf{C}$. The spectral behaviour of the EQE then follows the absorption spectrum within the active layer modified by any cavity interference effects. Charge collection-narrowed OPD (c,d): using a thick bulk heterojunction photo-active layer, shorter wavelength photons ( $\mathbf{A}$ and $\mathbf{B}$ ) are absorbed near the indium tin oxide (ITO) side, therefore the extraction of photo-generated electrons is hindered. In this case, the charge collection efficiency, $\eta_{\text {colll }}$, is spectrally narrowed to only the wavelengths that are near the onset of the absorption (C) where the junction extinction coefficient is low. The EQE therefore peaks sharply at the onset of the absorption. 
Charge collection-narrowed OPDs. Figure $1 \mathrm{c}, \mathrm{d}$ shows a thick junction equivalent of Fig. 1a,b. In this case, the heterojunction is chosen to be much thicker than the inverse of the absorption coefficient $(\alpha)$ of the photo-active layer, that is, it has a large optical density $(\alpha d \gg 1)$. Since $\alpha$ can typically be of order $10^{5} \mathrm{~cm}^{-1}$ for organic semiconductor combinations, a photoactive layer thickness of $\sim 2 \mu \mathrm{m}$ will satisfy this condition. Under such circumstances, wavelengths $\mathbf{A}$ and $\mathbf{B}$ above the optical gap are very strongly absorbed and produce an exponential photocarrier distribution profile according to the Beer-Lambert Law. There are no interference effects since no light reaches the back electrode, and one observes surface photo-carrier generation near the semi-transparent electrode. However, for light absorption at wavelengths near the optical gap (C), light can penetrate through the photo-active layer and volume photo-carrier generation occurs with the associated influence of optical interference.

Consider now the relative charge collection efficiencies for the two cases, that is, the surface generation near the anode (responsible for hole extraction in reverse bias) for $\mathbf{A}$ and $\mathbf{B}$ (above-gap) wavelengths and volume generation for $\mathbf{C}$ (near-orsub gap). When electrons and holes are photo-generated close to the anode as for $\mathbf{A}$ and $\mathbf{B}$ (the hole-extracting electrode, usually ITO), charge carrier drift preferentially extracts holes with short transit times ${ }^{41}$. Electrons must traverse the entire thick junction and therefore have much longer transit times. The diode displays strongly imbalanced charge carrier transport and negative space charge accumulates near the anode as a result of delayed electron extraction ${ }^{43}$. The space charge screens the electric field responsible for extracting the carriers (including holes). Consequently electrons and holes recombine and charge carrier collection is suppressed. This behaviour is akin to a shallow Schottky diode comprising a $p$-type semiconductor-the electric field is screened near the high work function electrode (ITO) in a direct analogy with the recombination (field free) zone in Schottky diodes ${ }^{44}$. The situation is very different for volume photogeneration $(\mathbf{C})$ where the transport is more balanced. In a perfectly balanced diode, electrons and holes are extracted with the same efficiency preventing the formation of space charge. Hence, for wavelengths near the absorption onset, the collection efficiency is high, and for shorter wavelengths it is poor (in the limit zero) although a portion of photo-generated carriers (at C) can also be lost due to the charge recombination near the anode. This imposes a strong wavelength dependence on the collection efficiency, which dominates the EQE as shown in Fig. 1d. The peak wavelength (and bandwidth) of the EQE can then be finetuned by adjusting the junction thickness as will now be demonstrated. We call this concept charge collection narrowing $(\mathrm{CCN})$. By increasing the reverse bias voltage, the charge carrier transit time becomes shorter and that in turn decreases the formation of space charges for surface generated carriers. This causes the spectral selectivity to diminish at large reverse bias operating voltages. This is shown in Supplementary Fig. 1. It is important to emphasize that these CCN OPDs can deliver high responsivity, low noise and sufficient speed at reverse bias voltages $<2 \mathrm{~V}$, where the narrowband spectral response is conserved. Low operating voltages are of course favoured because they deliver circuit simplicity and low power consumption. This sensitivity to the applied voltage re-affirms the basic concept of $\mathrm{CCN}$.

It is important to note that the thicknesses required for achieving the $\mathrm{CCN}$-derived narrowband responses (microns) are much larger than those previously used for broadband organic semiconductor photodetectors $(700 \mathrm{~nm})$ that can still be considered as electrically thin ${ }^{35}$. The transport and optical properties of typical organic semiconductor donor:acceptor blends enable the large thicknesses used in CCN OPDs to guarantee surface charge generation for wavelengths outside the desired spectral range and volume charge generation for the required wavelengths. This vital condition is demonstrated by way of optical simulations in Supplementary Fig. 2, which compares the charge generation distribution profiles in $700-$ and $2,000-\mathrm{nm}$ thick PCDTBT:PC70BM junctions.

Experimental results. PCDTBT:PC70BM and poly[2,5-(2-octyldodecyl)-3,6-diketopyrrolopyrrole-alt-5,5-(2,5-di(thien-2-yl)thieno [3,2-b]thiophene)] (DPP-DTT):PC70BM BHJ systems were used to construct the CCN OPDs. These materials are archetypal high efficiency donor:acceptor combinations in organic photovoltaics ${ }^{45}$ with their molecular structures shown in Fig. 2. Both donor polymers when blended with PC70BM are broadband light absorbers and DPP-DTT has recently been introduced as a high

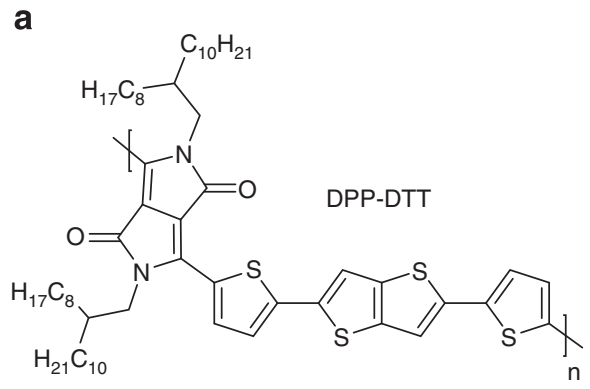

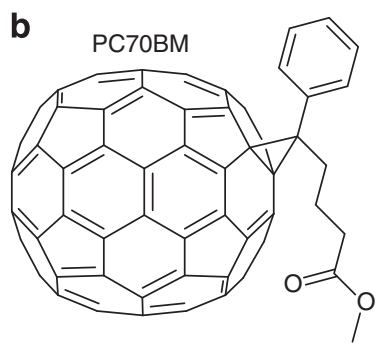

C

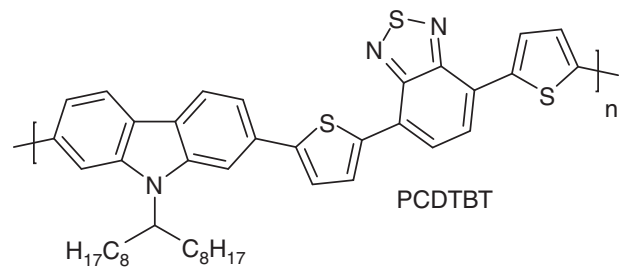

Figure 2 | Molecular structures of the semiconductors used in this work. (a) DPP-DTT (electron donor) (b) PC7OBM (electron acceptor) and (c) PCDTBT (electron donor). 
mobility polymer for field effect transistors ${ }^{46}$. The PCDTBT: PC70BM heterojunction has an absorption onset at $\sim 650 \mathrm{~nm}$ and DPP-DTT is a narrow optical-gap polymer with an absorption edge at $\sim 950 \mathrm{~nm}$ (Fig. 3a). Using high molecular weight polymers $\left(\bar{M}_{\mathrm{w}}=122 \mathrm{kDa}\right.$, polydispersity index (PDI) $=5.4$ for PCDTBT and $\bar{M}_{\mathrm{w}}=350 \mathrm{kDa}, \mathrm{PDI}=2.8$ for DPP-DTT) and appropriate solution thermal treatments, high solution viscosities enable the fabrication of thick, uniform films by spin coating ${ }^{47}$. Based on the thick junction methodology described above and an understanding of

a

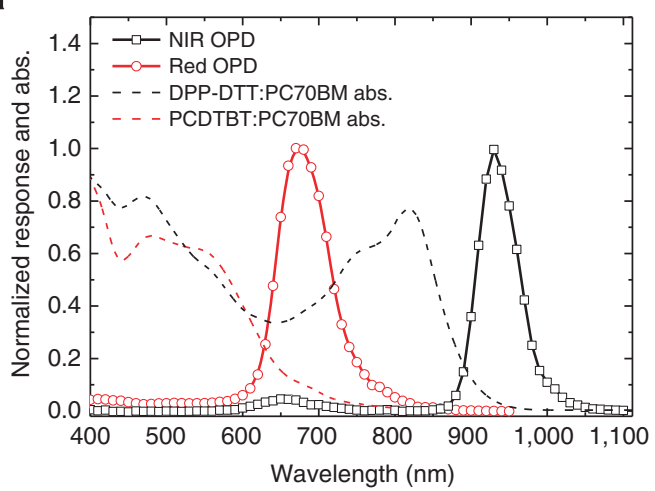

b

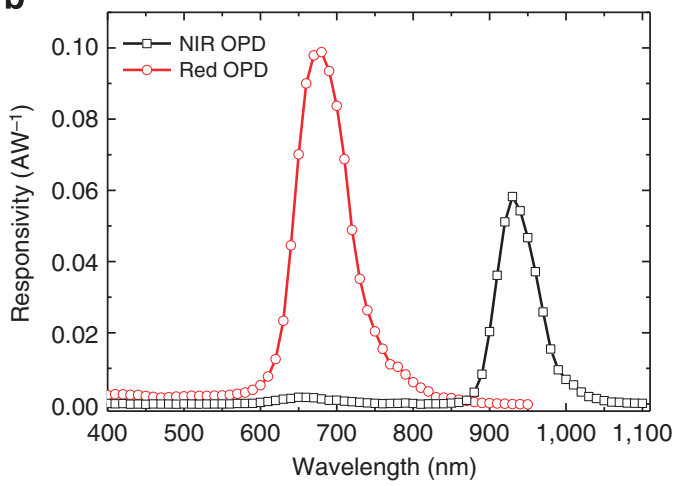

c

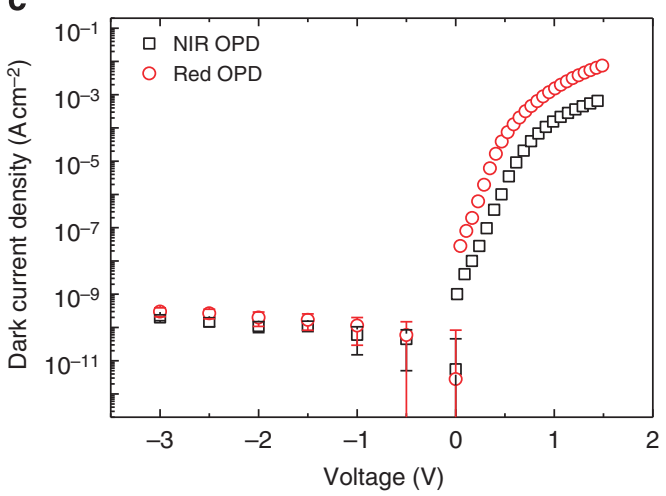

Figure 3 | Spectral dark current density-voltage response of the CCN red and NIR OPDs. (a) The normalized EQE of the narrowband CCN OPDs comprising thick junctions of PCDTBT (red) and DPP-DTT (NIR) blended with PC7OBM. The normalized absorption of PCDTBT:PC7OBM and DPPDTT:PC7OBM blends are also shown (dashed lines). The photoresponse peaks at the absorption edge as predicted under the $\mathrm{CCN}$ regime. (b) The responsivity of the OPDs under reverse bias voltage of $1 \mathrm{~V}$. (c) Dark current density-voltage $(J-V)$ curves for the CCN OPDs of (a) and (b). In forward bias, the dark current is measured by a normal $J-V$ sweep but in reverse bias the extremely low dark current, means a time average of the current is taken for all points and the standard deviation (s.d.) of the fluctuations is specified by the error bars. how to further suppress the dark current with hole-and-electronblocking layers ${ }^{35,48}$, we arrived at the following optimized CNN, thick junction OPD structures: NIR OPD-ITO $80 \mathrm{~nm} /$ PEDOT:PSS $20 \mathrm{~nm} / \mathrm{DPP}-\mathrm{DTT}: P C 70 \mathrm{BM} \quad 2 \mu \mathrm{m} / \mathrm{C} 60 \quad 50 \mathrm{~nm} / \mathrm{Al}$ $100 \mathrm{~nm}$ and Red OPD-ITO $80 \mathrm{~nm} /$ PEDOT:PSS $20 \mathrm{~nm} /$ PCDTBT:PC70BM $2 \mu \mathrm{m} / \mathrm{C} 6050 \mathrm{~nm} / \mathrm{Al} 100 \mathrm{~nm}$. The normalized absorption spectra for the two blend systems (thin films on glass) and the normalized EQE of the OPDs are shown in Fig. 3a. Figure $3 \mathrm{~b}$ shows the corresponding responsivity spectra (measured at $-1 \mathrm{~V}$ ). Both red and NIR CCN OPDs have FWHM $<90 \mathrm{~nm}$.

\section{Discussion}

The dark current of OPDs under reverse bias theoretically depends on the electronics of the system ${ }^{49}$. However, the large density of defects (surface roughness, particles and ITO spikes) in solution-processed organic semiconductor junctions normally dominates the dark current, particularly in large area devices 34 . Use of a thick junction markedly decreases the defect density ${ }^{35}$. Figure $3 \mathrm{c}$ shows the dark current of the two CCN OPDs. In both cases, the dark current (under reverse bias) is extremely low, for example, at an operating reverse bias voltage of $1 \mathrm{~V}$ the dark currents are $<10^{-10} \mathrm{~A}$.

Although the dark current $\left(i_{\mathrm{d}}\right)$ is an indicator of the noise figure of merit for photodetectors, one cannot directly define the noise current and specific detectivity via shot noise, which is proportional to the square root of the dark current ${ }^{50}$. In fact, the inferred shot noise, which is due to fluctuations in the Poissonian distributed quanta of the current (that is, electrons) obtained from the dark current $\left(\sqrt{2 e i_{\mathrm{d}} B}\right)$, where $e$ is the unit charge and $B$ is the detection bandwidth (inversely proportional to the detection time constant), has been shown to differ from the real, measured noise even when the thermal noise is negligible $17,19,51,52$. If we use the shot noise to evaluate the noise equivalent power and the specific detectivity $D^{*}$, we obtain a larger value for $D^{*}$, of the order $>10^{13}$ Jones. However, by measuring the noise spectral density from the Fourier transform of the current versus time (see Supplementary Fig. 3), a more reliable value for the detectivity can be calculated from the noise power spectrum. In this case, the detectivity can be expressed as ${ }^{34}$ :

$$
D^{*}=\frac{\lambda e \sqrt{A} \cdot \mathrm{EQE}}{h c i_{\text {noise }}}:\left(\sqrt{\mathrm{Hz}} \mathrm{cmW}^{-1}\right)
$$

where $\lambda$ is the detection wavelength, $A$ the device area, $h=$ Planck's constant, $c=$ speed of light and $i_{\text {noise }}$ the noise current spectral density (with unit of $\mathrm{AHz}^{-1 / 2}$ ). The $i_{\text {noise }}$ can be read from the noise spectrum at the detection frequency at which the EQE is measured (see Supplementary Fig. 3). Figure 4 shows the specific detectivities $D^{*}$ for the red and NIR CCN OPDs evaluated at $120 \mathrm{~Hz}$, where the EQE was measured; these values are comparable within OPDs, whose specific detectivities range from between $10^{9}$ to $10^{12}$ at room temperature ${ }^{2,53}$. These results also demonstrate that our CCN OPDs are visible-blind, that is, there essentially is no contribution to the photocurrent from visible wavelengths. We note there is a minor contribution to the EQE and detectivity $(<5 \%)$ at $650 \mathrm{~nm}$ in our NIR CCN OPDs due to a minimum in the DPP-DTT absorption at that wavelength.

The LDR and frequency bandwidth are also critical performance parameters for photodiodes as they represent the light intensity operating range and temporal response of the device, respectively. For the red OPD, we observed 8 orders of magnitude linearity in the photoresponse (corresponding to either $80 \mathrm{~dB}$ or $160 \mathrm{~dB}$ following refs $51,52,54$ and 2 , respectively) and a $-3 \mathrm{~dB}$ cut-off frequency of $100 \mathrm{kHz}$ at $-1 \mathrm{~V}$ (Fig. 5). Due to light source limitations, we were not able to measure the frequency response 
and LDR for the NIR CCN OPD. However, because the charge carrier mobility of DPP-DTT is at least 3 orders of magnitude larger than PCDTBT ${ }^{41,46}$, one would expect an LDR and $-3 \mathrm{~dB}$ frequency response at least as good as the PCDTBT:PC70BM devices.

Finally, and as indicated above, the peak EQE and hence the chosen spectral range of the CCN OPDs depends on the bulk heterojunction thickness. By increasing the junction thickness, wavelengths with only lower absorption cross-sections can penetrate to the cathode. Increasing the thickness thus causes longer wavelengths to contribute to surface charge carrier generation and this therefore shifts the response peak to longer wavelengths. In Fig. 6, the EQE spectra for PCDTBT:PC70BM CCN OPDs with junction thicknesses of $1.5,2$ and $3 \mu \mathrm{m}$ are shown as well as the absorption coefficient of the blend. The EQE can be fine-tuned over a $100 \mathrm{~nm}$ range through the absorption edge of the PCDTBT:PC70BM blend. It is however worth noting that the $\mathrm{EQE}$ does decrease as one tunes the spectral response towards the absorption edge of the blend. This arises due to less light absorption within the active layer relative to the parasitic absorption in the aluminium cathode (Supplementary Fig. 4) as the wavelength increases. Further losses in CCN OPDs are associated with recombination of those charge carriers generated by the desired wavelengths near the anode (Supplementary Fig. 2). However, it is important to note that the extraordinarily

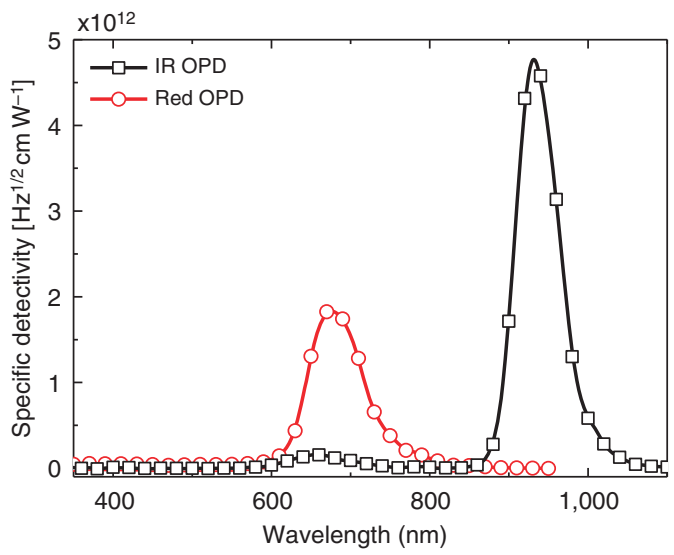

Figure 4 | Specific detectivity of red and NIR CCN OPDs. The specific detectivity is calculated based on the measured EQE (at $120 \mathrm{~Hz}$ ) and the actual measured noise current under a reverse bias voltage of $1 \mathrm{~V}$. low dark current and noise of the thick junctions provide the possibility of amplification of photocurrent, which is then electronically manifested as a large specific detectivity.

In conclusion, we introduce the novel concept of CCN OPDs in a thick bulk heterojunction architecture. The CCN physics allows for precise tailoring of the spectral shape of the IQE. We demonstrate the first visible-blind sub-100 nm FWHM red and NIR photodetectors without the use of input filtering. The use of a thick junction decreases the defect density and therefore significantly suppresses the dark current resulting in high specific detectivities $\left(\sim 10^{12} \mathrm{~J}\right)$ and LDR. Moreover, we demonstrate that these CCN OPDs can be spectrally fine-tuned by $>100 \mathrm{~nm}$. This methodology is generic and the detection window can be chosen depending on the absorption onset of the system.

The wavelength selectivity of CCN narrowband OPDs is limited by the absorption onset of the donor and acceptor combination. If fullerenes are used, then the detection wavelengths possible using CCN are $>600 \mathrm{~nm}$. Hence, to realize green and blue narrowband photodiodes wide optical-gap acceptors are required and this remains both a challenge and opportunity in the organic solar cell and photodiode community.

\section{Methods}

Materials. PCDTBT $\left(\bar{M}_{\mathrm{w}}=122 \mathrm{kDa}\right.$, PDI $\left.=5.4\right)$ was purchased from SJPC, Canada. The molecular weight of the PCDTBT was determined with gel permeation chromatography in 1,2,4-trichlorobenzene at $135^{\circ} \mathrm{C}$. PC70BM and C60 were purchased from American Dye Source Inc., Canada and used without further purification. DPP-DTT $\left(\bar{M}_{\mathrm{w}}=350 \mathrm{kDa}, \mathrm{PDI}=2.8\right)$ was synthesized by the Institute of Materials Research and Engineering (IMRE) Singapore based on the methodology previously described ${ }^{46}$.

Sample preparation. The glass substrates with pre-etched ITO for bulk heterojunction OPDs were purchased from Kintec and mechanically cleaned by scrubbing using a soft cloth in a $90^{\circ} \mathrm{C}$ warm Alconox (detergent) solution. Cleaning was followed by sequential ultrasonication in Alconox, de-ionized water, acetone and 2-propanol. After drying the substrates under a nitrogen flow, a 30-nm thick poly(3,4-ethylenedioxythiophene):poly(styrene sulfonate) (PEDOT:PSS; Baytron P VPAl4083) film was spin-coated at 5,000 r.p.m.. The PEDOT:PSS layer was baked at $170^{\circ} \mathrm{C}$ for $10 \mathrm{~min}$ in air. All the device edges were cleaned with a wet cloth to prevent lateral current leakage through the PEDOT:PSS. Solutions of PCDTBT and DPP-DTT were prepared in 1,2-dichlorobenzene by stirring at $150^{\circ} \mathrm{C}$ for an hour at a concentration of $10 \mathrm{mg} \mathrm{ml}^{-1}$. Solutions were then slowly cooled to $80^{\circ} \mathrm{C}$ and added to PC70BM in a weight ratio of $1: 4$ or $1: 3$ then stirred for $6 \mathrm{~h}$ at $80^{\circ} \mathrm{C}$. Solutions $\left(\right.$ at $80^{\circ} \mathrm{C}$ ) were then dispensed on the cold substrate. The fast cooling of the solution on dispensing on the cold surface results in a dramatic increase in the solution viscosity as shown by Wolfer et al. ${ }^{47}$ The substrates were spun at 400 r.p.m. for $>30 \mathrm{~min}$ followed by slow drying over $6 \mathrm{~h}$ at $40^{\circ} \mathrm{C}$. A $50 \mathrm{~nm}$ layer of C60 was deposited by thermal evaporation under a vacuum of $\sim 10^{-6} \mathrm{mbar}$ followed by $100 \mathrm{~nm}$ of aluminium deposited under the same vacuum conditions to complete the devices. The resulting device areas were $0.2 \mathrm{~cm}^{2}$ with six devices per substrate.
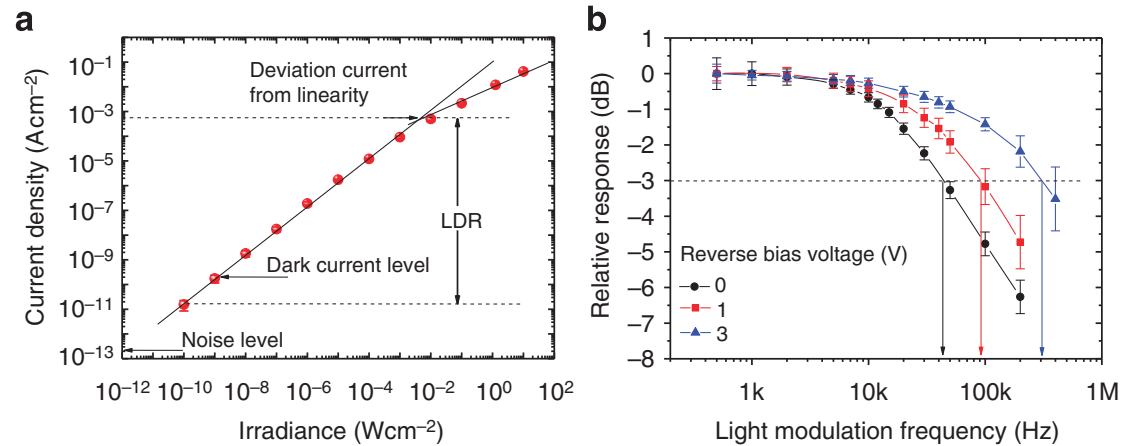

Figure 5 | Frequency and intensity response of the CCN red OPD. (a) Linear dynamic range (LDR) for the red OPD measured at $-1 \mathrm{~V}$ (pump wavelength $\sim 650 \mathrm{~nm}$ ). The photodiode response is linear over 8 orders of magnitude. The error bars represent the s.d. from the mean value of six measurements. (b) Normalized response loss of the red OPD versus the input signal frequency (light modulation frequency at an intensity of $\sim 1 \mathrm{~mW} \mathrm{~cm}^{-2}$ ). The $-3 \mathrm{~dB}$ point is specified with the dashed line. The error bars correspond to the s.d. from the mean value of the signal versus time. 
a

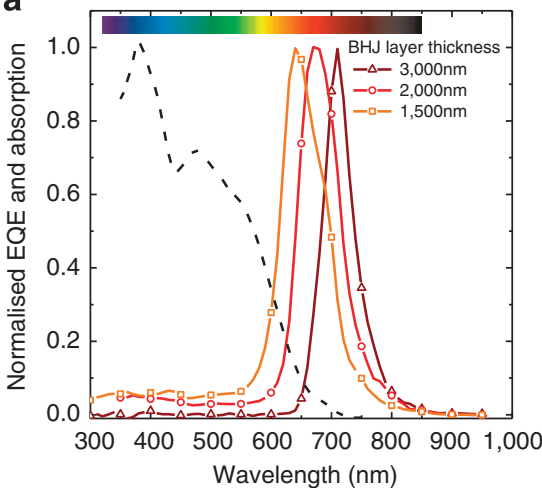

b

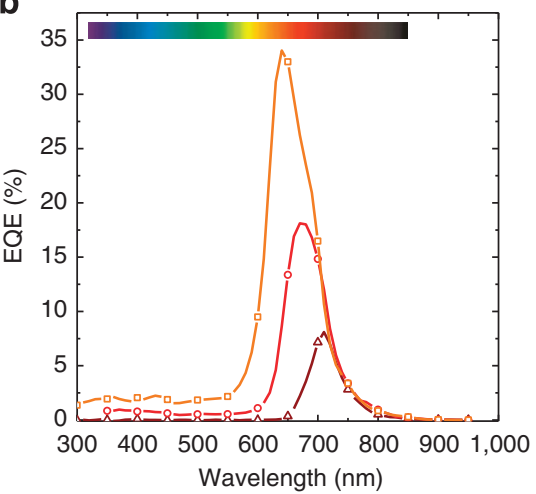

Figure 6 | The tunability of the CCN OPDs. The normalized EQE (a) and EQE (b) of PCDTBT:PC7OBM photodiodes measured at $120 \mathrm{~Hz}$ and $-1 \mathrm{~V}$ with different thicknesses allowing for fine-tuning the charge carrier photogeneration profile and thereby the EQE. As the active layer thickness increases from 1,500 to $3,000 \mathrm{~nm}$ the photogeneration in the volume occurs at longer wavelengths and the EQE peak red shifts from red to the NIR. The dashed line represents the absorption coefficient of the blend.

EQE and optical simulations. EQEs were measured using a PV Measurements Inc. QEX7 set-up at $120 \mathrm{~Hz}$. The optical field distributions in the photodiodes were simulated using a computer code based on the transfer matrix method developed by van de Lagemaat and co-workers ${ }^{44}$ from the National Renewable Energy Laboratory (NREL, USA). For these simulations optical constants (refractive index and extinction coefficients) of the all the materials in the structure are required. In this regard, we performed spectroscopic ellipsometry (SE) measurements for PCDTBT:PC70BM films on silicon wafers (Supplementary Fig. 5; used for transfer matrix simulations in Fig. 1). Optical constants of the other layers have been measured previously ${ }^{30}$. The SE measurements were performed with a J.A. Woollam ultraviolet-variable angle spectroscopic ellipsometer (VUV-VASE; GEN II) with autoretarder. J.A. Woollam WVASE32 software was used to fit the data.

Dark/photocurrent and LDR measurement. Dark currents and photocurrents were measured using an Agilent semiconductor analyzer B1500A by averaging the current over time for each voltage step. The devices were mounted on an optical table in a Faraday cage with a small aperture for the laser beam. Triax leads were used to minimize the measurement noise. An ITO on glass panel that was electrically connected to the cage by silver paste to further reduce the electrical noise covered the aperture. A diode laser operating continuously at $650 \mathrm{~nm}$ (Laserver) was used as the illumination source with a series of neutral density filters purchased from Thorlabs and Holmarc for the LDR measurements.

Temporal response. Frequency dependent measurements were made using a Nichia red LED modulated by using an arbitrary wave generator (Agilent 33250A). The photocurrent response of the photodiode was recorded using a digital storage oscilloscope (LeCroy Waverunner A6200).

\section{References}

1. Baeg, K.-J., Binda, M., Natali, D., Caironi, M. \& Noh, Y.-Y. Organic light detectors: photodiodes and phototransistors. Adv. Mater. 25, 4267-4295 (2013).

2. Gong, X. et al. High-detectivity polymer photodetectors with spectral response from $300 \mathrm{~nm}$ to $1450 \mathrm{~nm}$. Science 325, 1665-1667 (2009).

3. Lukac, R. Single-sensor imaging in consumer digital cameras: a survey of recent advances and future directions. J. Real-Time Image Processing 1, 45-52 (2006).

4. Nishiwaki, S., Nakamura, T., Hiramoto, M., Fujii, T. \& Suzuki, M.-A. Efficient colour splitters for high-pixel-density image sensors. Nat. Photonics 7, 240-254 (2013).

5. Park, H. et al. Filter-free image sensor pixels comprising silicon nanowires with selective color absorption. Nano Lett. 14, 1804-1809 (2014).

6. Jansen-van Vuuren, R. D., Pivrikas, A., Pandey, A. K. \& Burn, P. L. Colour selective organic photodetectors utilizing ketocyanine-cored dendrimers. J. Mater. Chem. C 1, 3532-3543 (2013).

7. Gautam, V., Bag, M. \& Narayan, K. S. Single-pixel, single-layer polymer device as a tricolor sensor with signals mimicking natural photoreceptors. J. Am. Chem. Soc. 133, 17942-17949 (2011).

8. Yusuke, H., Kyu-Sik, K., Hyeon-Gu, J. \& Musubu, I. Enhancing spectral contrast in organic red-light photodetectors based on a light-absorbing and exciton-blocking layered system. J. Appl. Phys. 108, 034502 (2010).

9. Jansen-van Vuuren, R. D. et al. Determining the absorption tolerance of single chromophore photodiodes for machine vision. Appl. Phys. Lett. 96, 253303 (2010).
10. Rauch, T. et al. Near-infrared imaging with quantum-dot-sensitized organic photodiodes. Nat. Photonics 3, 332-336 (2009).

11. Ohmori, Y. et al. Realization of polymeric optical integrated devices utilizing organic light-emitting diodes and photodetectors fabricated on a polymeric waveguide. IEEE J. Sel. Top. Quantum Electron. 10, 70-78 (2004).

12. Antognazza, M. R., Scherf, U., Monti, P. \& Lanzani, G. Organic-based tristimuli colorimeter. Appl. Phys. Lett. 90, 163509-163512 (2007).

13. Morimune, T., Kajii, H. \& Ohmori, Y. Photoresponse properties of a high-speed organic photodetector based on copper-phthalocyanine under red light illumination. Photonics Technol. Lett. 18, 2662-2664 (2006).

14. Li, L., Huang, Y., Peng, J., Cao, Y. \& Peng, X. Highly responsive organic near-infrared photodetectors based on a porphyrin small molecule. J. Mater. Chem. C 2, 1372-1375 (2014).

15. Arca, F., Sramek, M., Tedde, S. F., Lugli, P. \& Hayden, O. Near-infrared organic photodiodes. IEEE J. Quant. Electron. 49, 1016-1025 (2013).

16. Dong, H., Zhu, H., Meng, Q., Gong, X. \& Hu, W. Organic photoresponse materials and devices. Chem. Soc. Rev. 41, 1754-1808 (2012).

17. Dong, R. et al. An Ultraviolet-to-NIR Broad Spectral Nanocomposite Photodetector with Gain. Adv. Opt. Mater. 2, 549-554 (2014).

18. Chen, H.-Y., Lo, M. K. F., Yang, G., Monbouquette, H. G. \& Yang, Y. Nanoparticle-assisted high photoconductive gain in composites of polymer and fullerene. Nat. Nanotechnol. 3, 543-547 (2008).

19. Yao, Y. et al. Plastic Near-infrared photodetectors utilizing low band gap polymer. Adv. Mater. 19, 3979-3983 (2007).

20. Soci, C. et al. Photoconductivity of a low-bandgap conjugated polymer. Adv. Funct. Mater. 17, 632-636 (2007).

21. Vandewal, K. et al. Efficient charge generation by relaxed charge-transfer states at organic interfaces. Nat. Mater. 13, 63-68 (2014).

22. Yang, C.-M. et al. Infrared photocurrent response of charge-transfer exciton in polymer bulk heterojunction. Appl. Phys. Lett. 92, 083504 (2008).

23. Li, G. et al. High-efficiency solution processable polymer photovoltaic cells by self-organization of polymer blends. Nat. Mater. 4, 864-868 (2005).

24. Kong, H. et al. Solution processable organic $\mathrm{p}-\mathrm{n}$ junction bilayer vertical photodiodes. Organ. Electron. 14, 703-710 (2013).

25. Zhang, Y. et al. Channel II photocurrent quantification in narrow optical gap polymer-fullerene solar cells with complimentary acceptor absorption. Appl. Phys. Lett. 102, 223302 (2013).

26. Sonar, P., Fong Lim, J. P. \& Chan, K. L. Organic non-fullerene acceptors for organic photovoltaics. Energy Environ. Sci. 4, 1558-1574 (2011).

27. Cook, S. et al. A photophysical study of PCBM thin films. Chem. Phys. Lett. 445, 276-280 (2007).

28. Aihara, S. et al. Stacked Image sensor with green- and red-sensitive organic photoconductive films applying zinc oxide thin-film transistors to a signal readout circuit. IEEE Trans. Electron. Dev. 56, 2570-2576 (2009).

29. Armin, A. et al. Quantum efficiency of organic solar cells: electro-optical cavity considerations. ACS Photonics 1, 173-181 (2014).

30. Lyons, D. M. et al. Narrow band green organic photodiodes for imaging. Org. Electron. 15, 2903-2911 (2014).

31. Lupton, J. M. et al. Organic microcavity photodiodes. Adv. Mater. 15, 1471-1474 (2003).

32. An, K. H., O’Connor, B., Pipe, K. P. \& Shtein, M. Organic photodetector with spectral response tunable across the visible spectrum by means of internal optical microcavity. Org. Electron. 10, 1152-1157 (2009).

33. Harrison, M., Grüner, J. \& Spencer, G. Analysis of the photocurrent action spectra of MEH-PPV polymer photodiodes. Phys. Rev. B 55, 7831-7849 (1997). 
34. Agostinelli, J. A., Kowarz, M. W. \& Liao, L.-S. Passive matrix oled display having increased size. US patent 20060091794 (2006).

35. Armin, A. et al. Thick junction broadband organic photodiodes. Laser Photonics Rev. 8, 924-932 (2014).

36. Peumans, P., Yakimov, A. \& Forrest, S. R. Small molecular weight organic thinfilm photodetectors and solar cells. J. Appl. Phys. 93, 3693-3723 (2003).

37. Armin, A., Zhang, Y., Burn, P. L., Meredith, P. \& Pivrikas, A. Measuring internal quantum efficiency to demonstrate hot exciton dissociation. Nat. Mater. 12, 593-593 (2013).

38. Dimitrov, S. D. et al. Towards optimisation of photocurrent from fullerene excitons in organic solar cells. Energy Environ. Sci. 7, 1037-1043 (2014).

39. Mescher, J., Christ, N., Kettlitz, S., Colsmann, A. \& Lemmer, U. Influence of the spatial photocarrier generation profile on the performance of organic solar cells. Appl. Phys. Lett. 101, 073301 (2012).

40. Burkhard, G. F., Hoke, E. T. \& McGehee, M. D. Accounting for interference, scattering, and electrode absorption to make accurate internal quantum efficiency measurements in organic and other thin solar cells. Adv. Mater. 22, 3293-3297 (2010).

41. Armin, A. et al. Balanced carrier mobilities: not a necessary condition for highefficiency thin organic solar cells as determined by MIS-CELIV. Adv. Energy Mater. 4, 1300954 (2014).

42. Jamieson, F. C., Agostinelli, T., Azimi, H., Nelson, J. \& Durrant, J. R. Fieldindependent charge photogeneration in PCPDTBT/PC70BM solar cells. J. Phys. Chem. Lett. 1, 3306-3310 (2010).

43. Kirchartz, T., Agostinelli, T., Campoy-Quiles, M., Gong, W. \& Nelson, J. Understanding the thickness-dependent performance of organic bulk heterojunction solar cells: the influence of mobility, lifetime, and space charge. J. Phys. Chem. Lett. 3, 3470-3475 (2012).

44. Morfa, A. J., Nardes, A. M., Shaheen, S. E., Kopidakis, N. \& van de Lagemaat, J. Time-of-flight studies of electron-collection kinetics in polymer:fullerene bulkheterojunction solar cells. Adv. Funct. Mater. 21, 2580-2586 (2011).

45. Li, W. et al. Efficient small bandgap polymer solar cells with high fill factors for $300 \mathrm{~nm}$ thick films. Adv. Mater. 25, 3182-3186 (2013).

46. Li, J. et al. A stable solution-processed polymer semiconductor with record high-mobility for printed transistors. Sci. Rep. 2, 745-1 (2012).

47. Wolfer, P. et al. Solution structure: defining polymer film morphology and optoelectronic device performance. J. Mater. Chem. C 2, 71-77 (2014).

48. Valouch, S. et al. Solution processed small molecule organic interfacial layers for low dark current polymer photodiodes. Org. Electron. 13, 2727-2732 (2012).

49. Wetzelaer, G. A. H., Kuik, M., Lenes, M. \& Blom, P. W. M. Origin of the darkcurrent ideality factor in polymer:fullerene bulk heterojunction solar cells. Appl. Phys. Lett. 99, 153506 (2011).

50. Büttiker, M. Scattering theory of current and intensity noise correlations in conductors and wave guides. Phys. Rev. B 46, 12485-12507 (1992).
51. Fang, Y., Guo, F., Xiao, Z. \& Huang, J. Large gain, low noise nanocomposite ultraviolet photodetectors with a linear dynamic range of $120 \mathrm{~dB}$. Adv. Opt. Mater. 2, 348-353 (2014).

52. Guo, F., Xiao, Z. \& Huang, J. Fullerene Photodetectors with a linear dynamic range of $90 \mathrm{~dB}$ enabled by a cross-linkable buffer layer. Adv. Opt. Mater. 1, 289-294 (2013).

53. Hamamatsu Photonics. Technical Information SD-12: Characteristics and use of Infrared Detectors. Catalogue No. KIRD9001E04 (Hamamatsu Photonics, Hamamatsu City, 2011).

54. Guo, F. et al. A nanocomposite ultraviolet photodetector based on interfacial trap-controlled charge injection. Nature Nanotechnol 7, 798-802 (2012).

\section{Acknowledgements}

A.A. was funded by University of Queensland International Scholarship. P.M. is an Australian Research Council Discovery Outstanding Research Fellow, and P.L.B. is a University of Queensland Vice Chancellor's Senior Research Fellow. N.K. acknowledges funding from the Energy Frontier Research Center 'Molecularly Engineered Energy Materials (MEEMs)' funded by the US Department of Energy, Office of Science, Office of Basic Energy Sciences under Contract Number DE-SC0001342:001. The work was part funded by the Australian Research Council Discovery Program (DP120103726). We acknowledge support from The University of Queensland (Strategic Initiative-Centre for Organic Photonics \& Electronics). This work was performed in part at the Australian National Fabrication Facility Queensland Node (ANFF-Q) - a company established under the National Collaborative Research Infrastructure Strategy to provide nano- and micro-fabrication facilities for Australia's researchers. We thank the Institute for Materials Research and Engineering (Singapore) for supplying the DPP-DTT.

\section{Author contributions}

A.A. prepared the samples and designed and performed the experiments and analysis. A.A., P.M. and N.K. developed the CNN concept. P.L.B. and P.M. supervised the project. All authors contributed to the interpretation of the results and preparation of the manuscript, which was drafted by A.A. and R.D.J.-vV. All authors have given approval to the final version of the manuscript.

\section{Additional information}

Supplementary Information accompanies this paper at http://www.nature.com/ naturecommunications

Competing financial interests: The authors declare no competing financial interests.

Reprints and permission information is available online at http://npg.nature.com/ reprintsandpermissions/

How to cite this article: Armin, A. et al. Narrowband light detection via internal quantum efficiency manipulation of organic photodiodes. Nat. Commun. 6:6343 doi: 10.1038/ncomms7343 (2015). 